\title{
Lattice QCD
}

\author{
Paul MACKENZIE*
}

Fermilab

\begin{abstract}
Modern lattice gauge theory calculations are making it possible for lattice QCD to play an increasingly important role in the quantitative investigation of the Standard Model. The fact that QCD is strongly coupled at large distances has required the development of nonperturbative methods and large-scale computer simulations to solve the theory. The development of successful numerical methods for QCD calculations puts us in a good position to be ready for the possible discovery of new strongly coupled forces beyond the Standard Model in the era of the Large Hadron Collider.
\end{abstract}

KEYWORDS: quantum chromodynamics, QCD, lattice gauge theory, quarks, gluons, strong interactions

\section{The Standard Model and beyond}

The Standard Model is amazingly successful (maddeningly so), yet its many gaps and puzzles make it clear that it is simply the low energy manifestation of new, higher-energy physics yet to be discovered. Its many parameters are simply arbitrary, having their observed values as a result of as yet undiscovered physics at higher energies than obtained up until now. Its three similar generations of fermions and three similar forces are undoubtedly related in some way yet to be discovered. To help understand what lies beyond the Standard Model, the fundamental parameters of the Standard Model are being pinned down with greater and greater precision in heavy flavor experiments around the world. New particles and forces are being sought in very high energy experiments at the Tevatron and will be soon at the LHC.

Lattice calculations are essential to this program in two ways. First, they are required to extract properties of quarks from properties of hadrons (particles that contain quarks). Unlike leptons, such as the electron or neutrino, quarks cannot be observed directly, but are confined permanently within hadrons. Their properties must be inferred using lattice gauge theory calculations.

Secondly, lattice gauge theory calculations are essential to prepare for possible new nonperturbative phenomena in coming experiments. Lattice gauge theory is the first and only general tool for solving nonperturbative quantum field theories. Of the four interactions known to particle physics, only one (quantum electrodynamics) is known to be described by a perturbative theory, whose properties can be expressed as a power series in the electromagnetic coupling

\footnotetext{
*E-mail address: mackenzie@fnal.gov
} 
constant, $\alpha_{\mathrm{em}}$, over all energy scales. Strong interactions are known to be described by a nonperturbative theory, quantum chromodynamics or QCD. String theory, the current best candidate for a theory of gravity, must have nonperturbative effects in it, or it would produce a space-time quite different from the four dimensional world that we live in. In the theory of the weak interactions, consider the "Higgs", the particle that generates particle masses. Is it

- an elementary, perturbative Higgs?

- a bound state of a new strong interactions (technicolor, topcolor, ...)?

- accompanied by very high energy gluino condensates (as in many models of supersymmetry with strongly coupled sectors)?

It is likely that whatever new physics is discovered by the Large Hadron Collider, it will contain some nonperturbative effects. QCD is providing an excellent test bed to sharpen our nonperturbative tools to prepare for such questions.

\section{Quarks, gluons, and lattice QCD}

Asymptotic freedom and quark confinement. In the early sixties, the classification properties of the observed hadrons led Gell-Mann and Zweig to note that the hadrons were arranged in multiplets as if they were composed of smaller particles, which Gell-Mann called quarks. In the same decade, deep inelastic scattering experiments at SLAC showed that in high energy electron-proton collisions, protons behaved as if they were composed of weakly interacting, almost-free constituents. Bjorken and Feynman called these entities partons. It was not immediately clear whether to regard quarks as actual particles, or whether they were merely a convenient classification tool. Furthermore, no one had ever seen a quark, so they seemed to be strongly confined inside hadrons. This seemed inconsistent with the weakly coupled nature observed in partons, so the relation between quarks and partons was not clear. Why should such almost-free constituents be permanently confined?

This paradox was resolved in 1973 with the discovery of the "asymptotic freedom" of QCD. The self-coupling of the gluons mediating the strong force caused the effective value of the strong coupling "constant" to become larger and larger at long distances (long compared with the proton radius), contrary to the well-known behavior of the electromagnetic coupling constant. This meant that even though the quarks were indeed weakly interacting at short distances and high energies, the force between them did not die off at long distances, leading to their permanent confinement. Gross, Politzer, and Wilczek shared the 2004 Nobel Prize for this discovery.

The consequence for particle physics is that, even though perturbation theory may be used to analyze quark-quark scattering at high energies, to infer the properties of quarks from the relatively low energy dynamics of hadron constituents, the nonperturbative methods of lattice QCD are required. 
Lattice gauge theory calculations. Quantum field theories are defined by their path integrals. For gauge theory, this may be written schematically as

$$
Z=\int d\left[A_{x \mu} \psi_{x}, \bar{\psi}_{x}\right] \exp (-S(A, \psi, \bar{\psi}))
$$

where $A$ and $\psi$ are the field variables of the gluons and quarks, and $S$ is the classical action of the theory. The quantum amplitude for a state of quarks and gluons at a given time to evolve into another state at a later time is obtained by integrating over all possible intervening classical field configurations. In principle, one integrates over independent fields defined at each space-time point. A quantum field theory is in principle defined by an infinite dimensional integral (not a very well-defined object). Quantum field theories must therefore be "regulated".

A lattice quantum field theory regulates the continuum theory by defining the fields on a four-dimensional space-time lattice. Quarks are defined on the sites of the lattice, and gluons on the links. Continuum quantum field theory is obtained in the zero lattice spacing limit. This limit is computationally very expensive, which is why large-scale computer simulations are required.

Operationally, lattice QCD calculations consist of several steps. First, sets of gauge configurations are computed that form a representative sample of the infinite set of possible configurations. They are constructed in long Markov chains with Monte Carlo methods, such as the venerable Metropolis method, or the more modern Hybrid Monte Carlo algorithm. Configurations are accumulated at several lattice spacings, and at several values of the masses of the light quarks in the fermi sea, which are heavier than the physical light quark masses. Final physics results must ultimately be extrapolated to the continuum and light quark mass limits.

Second, the propagation of quarks through the gauge configurations is calculated. This means solving the Dirac equation on each gauge configuration. On the lattice, this is a sparsematrix problem, solved with relaxation methods, such as the biconjugate gradient algorithm. This step can consume compute power that approaches that of the first step if many different physical processes are analyzed.

Third, hadron correlation functions and amplitudes are computed from the quark propagators. This is a computationally cheap step, consisting mostly of I/O.

State-of-the-art price/performance for computing hardware for this type of calculation is currently under $\$ 1 / \mathrm{MF}$. Larger projects are of order a few Teraflop-years. (That is, computing power of several delivered Teraflops, dedicated for a year.) Many types of large computers are used in lattice calculations, ${ }^{1)}$ such as the purpose-built QCDOC at Brookhaven National Laboratory (Fig. 1), large clusters of commodity computers such as the ones at Fermilab (Fig. 2) and many other places, and the IBM Blue Gene at KEK (Fig. 3), currently the largest computer in the world predominantly dedicated to lattice QCD calculations.

Progress in numerical science comes from both larger computers and from improvement of 


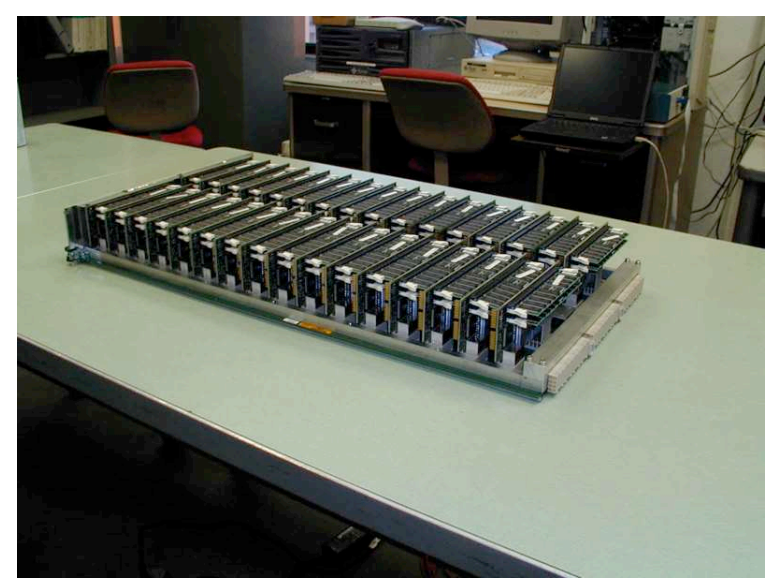

Fig. 1. A motherboard of the Columbia/Brookhaven QCDOC, a purpose built computer for lattice $\mathrm{QCD}$

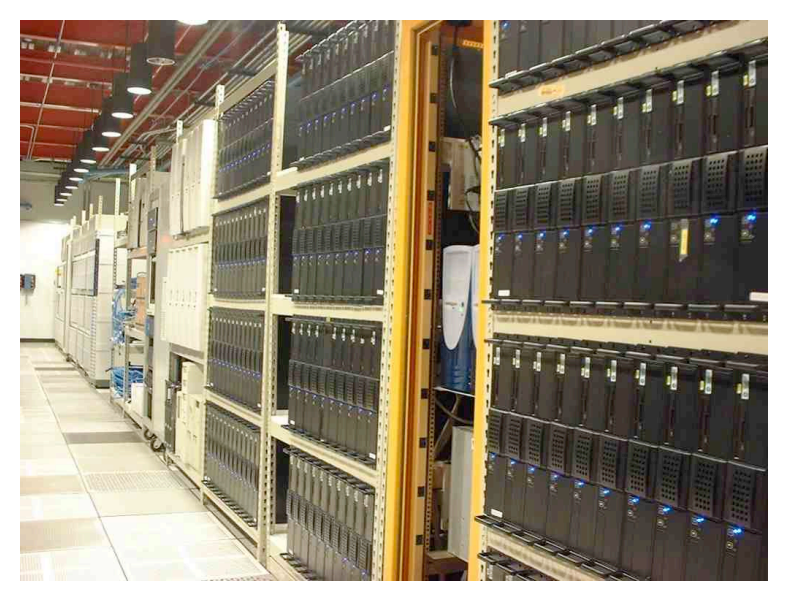

Fig. 2. Clusters at Fermilab devoted to lattice QCD.

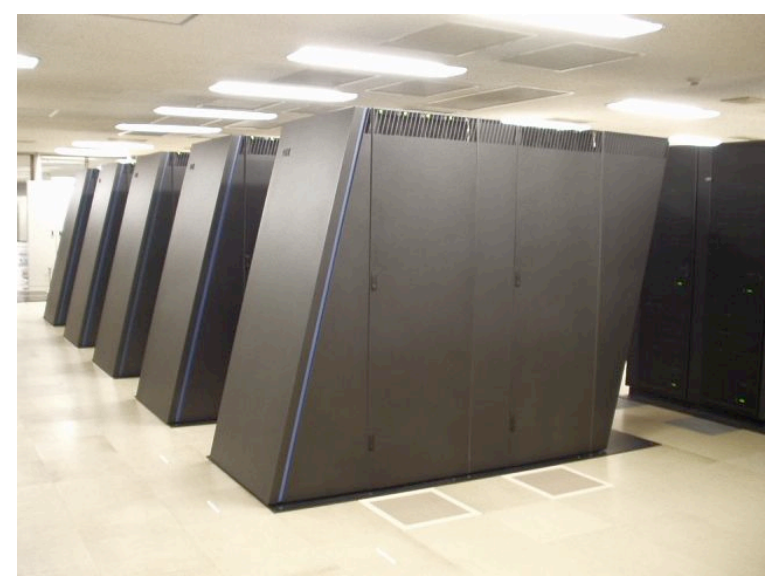

Fig. 3. The IBM Blue Gene computer at KEK, currently the largest computer predominantly dedicated to lattice QCD calculations. 
methods. A methodological improvement that has been particularly important for the work I will discuss is improved discretization. Numerical analysis tells us that if a derivative is approximated by a discrete difference, the resulting discretization errors vanish as the square of the lattice spacing:

$$
\frac{\partial \psi\left(x_{i}\right)}{\partial x}=\Delta_{x} \psi\left(x_{i}\right)+\mathcal{O}\left(a^{2}\right)
$$

where $\Delta_{x} \psi\left(x_{i}\right) \equiv\left(\psi\left(x_{i}+a\right)-\psi\left(x_{i}-a\right)\right) /(2 a)$. By incorporating next-to-nearest neighbor interactions, we can write down an approximation to the derivative whose errors vanish as a higher power of the lattice spacing:

$$
\frac{\partial \psi\left(x_{i}\right)}{\partial x}=\Delta_{x} \psi\left(x_{i}\right)-\frac{a^{2}}{6} \Delta_{x}^{3} \psi+\mathcal{O}\left(a^{4}\right) .
$$

This allows control of discretization errors with far less computing power than the simpler derivative.

It is relatively unambiguous how to remove the $\mathcal{O}\left(a^{2}\right)$ errors in the gluon action, ${ }^{2)}$ and the various improvements in use are closely related to each other. The situation is dramatically different with lattice fermions. There are several families of discretization methods, that each have very different virtues and drawbacks. Staggered fermions ${ }^{3-5)}$ can be calculated much more rapidly than the other methods. They have therefore been the first to produce reasonably precise unquenched results, and many of the results in Standard Model phenomenology in the next section use them. They have some ugly theoretical properties, ${ }^{6)}$ however, that lead some physicists to look at alternatives. Wilson fermions were the original fermions used introduced by Ken Wilson. They break chiral symmetry badly, and for that reason have had trouble getting to quark masses as light as the ones in nature. Recent algorithmic advances ${ }^{7-9}$ ) have altered this situation much for the better. Domain wall fermions ${ }^{10)}$ and overlap fermions ${ }^{11}$ ) have the nicest theoretical properties of all. They do not suffer the complications of staggered fermions, and have clean chiral structure unlike Wilson fermions. In the past, they have been by far the most expensive with which to calculate of all the methods, so phenomenological calculations are just beginning. Rapid algorithmic advance in the last few years have greatly sped up all the fermion methods, and it is not known at present which of these methods will ultimately prove superior. At present, lattice theorists around the world are hard at work on all of them, making sure that all methods give the same physical answers.

\section{Lattice QCD confronts experiment}

Progress in unquenched lattice QCD. In comparing QCD with experiment, we have several different types of tasks. One is comparing the results of QCD calculations with known experimental results and verifying that we can reproduce experiment. Since QCD is by now a very solidly established theory, this serves more to verify lattice gauge theory methods, rather than to test QCD. A second task is, where possible, to make predictions of physical results 
before they have been determined by experiment. Since hadron physics has been going on for decades, it is rare when opportunities can be found, but a few have been. A third, different type of task is to use verified lattice methods, combined with experiment, to obtain results that cannot be obtained by experiment alone. Examples of these include the quark masses and the strength of transformations of one quark to another under weak interactions (the Cabibbo-Kobayashi-Maskawa, or CKM, matrix elements). Since only hadrons are observed in experiment, and never quarks, these cannot be directly determined from experiment. Instead, quark masses and CKM matrix elements are used as parameters in lattice calculations and chosen so that the results of the calculations agree with experiment. These quark masses and CKM matrix elements are among the fundamental paramenters of the Standard Model that must ultimately be postdicted by future Beyond-the Standard-Model theories. Their determination is one of the most important tasks of lattice QCD as far as particle physics as a whole is concerned.

In the last few years, there has been dramatic progress in our ability to perform precise calculations of simple quantities. For twenty years after the first lattice Monte Carlo calculations appeared around 1980, almost all lattice QCD phenomenology was done in the quenched theory, meaning ignoring the effects of light quark-antiquark pairs. Although computationally much cheaper than correct unquenched calculations, this method introduces errors of unknown size into the results. The left-hand graph of Fig. 4 shows various combinations of particle masses and decay constants calculated in the quenched theory and shows around $10 \%$ discrepancies with experiment. There is no theory, however, that allows us to estimate in advance what size errors quenching introduces for any given quantity. Great strides, however, have been made in methods, in algorithms, and in computational power. They have now brought us to an era when unquenched calculations are becoming the norm, with all three light flavors of quark, $u, d$, and $s$, included dynamically.

The calculations in the graph of Fig. 4 were performed with improved staggered fermions (called "asqtad" fermions in the jargon). The right-hand graph of Fig. 4 shows the same quantities as on the left, but unquenched and now showing good agreement with experiment at the few percent level. ${ }^{12)}$ For these calculations, the masses of some quantities like the pion and kaon masses are used as inputs to fix the fundamental parameters of QCD, the quark masses and the strong coupling constant. Three different groups using this method, Fermilab, MILC, and HPQCD, then compared notes on their predictions for the simplest quantities they were calculating, with the results shown. These results are for the simplest physical quantities we know how to calculate in lattice QCD. The calculations are now being extended to more and more complicated quantities. Likewise, the results shown are obtained with staggered fermions, the least computationally costly of the fermion methods, and it will be interesting (and necessary)to verify that one obtains the same answers with more costly methods. 
The prediction of a particle mass: the $B_{c}$. Most of the particle masses and other simple quantities that are to be "predicted" by lattice QCD have been well known for fifty years, so that only postdiction is possible. An exception has been the mass of the $B_{c}$ meson, a meson made of a bottom quark and a charm antiquark. Bottom quarks were discovered only in the 1970's, and since they are rarely produced in association with charm quarks, $B_{c}$ mesons had not been observed as of a few years ago. Fig. 5 shows the predictions of unquenched lattice calculations, before the observation of the $B_{c} \cdot{ }^{13)}$ In December of 2004, the CDF experiment at Fermilab announced the discovery of the $B_{c}$. Their result for the mass is shown in the gold bar across the graph, in good agreement with the lattice prediction.

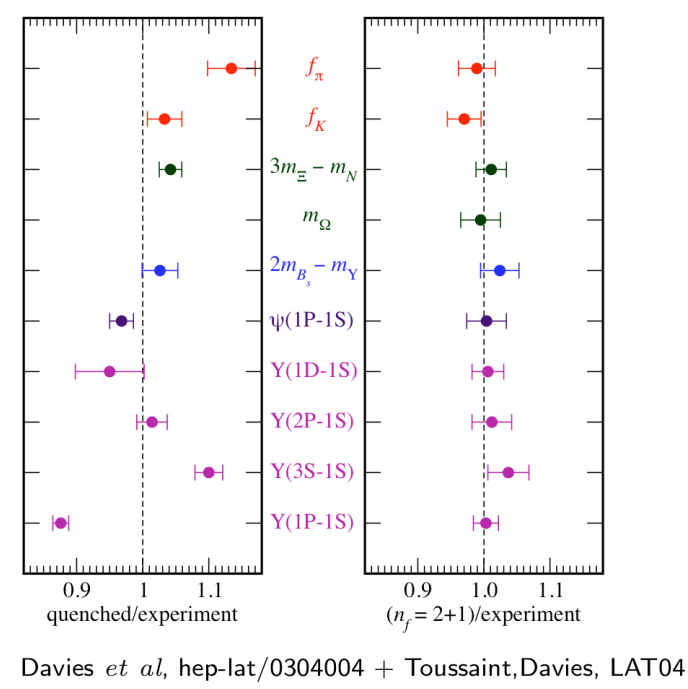

Fig. 4. Lattice predictions compared with experiment for simple quantities in quenched (left) and unquenched (right) lattice QCD. ${ }^{12)}$

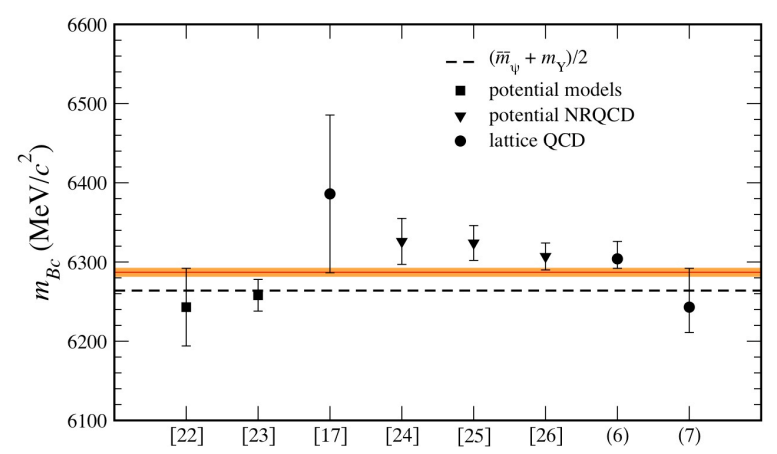

Fig. 5. The mass of the $B_{c}$ meson observed by the CDF collaboration (gold bar across the figure) compared with predictions of lattice QCD made before the observation (rightmost two data points). (Color online.) 


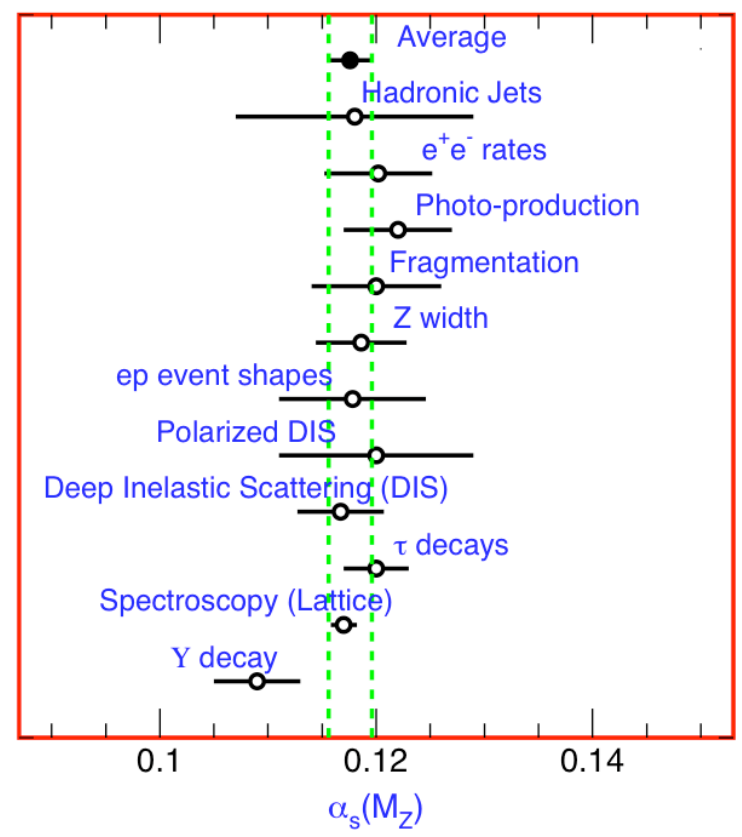

Fig. 6. Determinations of the strong coupling constant from a variety of high energy processes, evaluated by convention at the mass of the $Z$ boson, $M_{Z}$.(From the Review of Particle Properties. $)^{14)}$ The lattice determination (second from bottom) agrees well with the world average and is the most precise individual determination.

The strong coupling constant. The effective coupling "constant", $\alpha_{s}(E)$, governs the strength of the strong interactions of QCD. It is one of the best measured parameters of QCD. Asymptotic freedom means that $\alpha_{s}(E)$ is small in collisions at high energy, $E$. Therefor, perturbation theory can be used to analyze high energy collisions in terms of a power series in $\alpha_{s}(E)$. The strong coupling constant can be measured in a large number of high-energy processes, some of which are shown in the plot in Fig. 6. One can also obtain the strong coupling constant with lattice methods. One should obtain the same results if lattice methods are correct. One obtains $\alpha_{s}$ on the lattice by using it as a parameter in particle spectroscopy calculations, as in Ref. ${ }^{12)}$ One then uses perturbation theory to convert the lattice coupling constant to the form used in conventional continuum perturbation theory analyses. The result is shown in the next-to-bottom point in Fig. 6. It incorporates three-loop lattice perturbation theory. It agrees well with the world average of continuum results, as it should, and the lattice result is now the most precise of the individual determinations. The result is ${ }^{15)}$

$$
\alpha_{s}\left(M_{Z}\right)=0.1170(12) .
$$

The light quark masses. The value of the strong coupling constant was well known before lattice calculations. Its confirmation by lattice calculations is a welcome validation of lattice methods. By contrast, without the lattice, the values of the light quark masses can only be estimated approximately. The mass of the strange quark in particular plays an 
important role in analysis of weak interaction phenomenology, so a good determination of its value is a pressing concern. Quark models and a variety of phenomenological methods yielded conventional wisdom estimates of $m_{s} \sim 150 \mathrm{MeV}$ for the strange quark mass, and $m_{l} \sim 6 \mathrm{MeV}$ for the average of the up and down quark masses. That conventional wisdom, we now know, is far off the mark. With lattice QCD, we can determine these masses with firstprinciples calculations, for example, by tuning the quark masses to obtain the correct masses for pions and kaons. Last year, the MILC collaboration using improved staggered fermions reported $^{16,17)}$

$$
\begin{aligned}
& m_{s}=90(6) \mathrm{MeV}, \\
& m_{l}=3.3(3) \mathrm{MeV} .
\end{aligned}
$$

A recent paper by the CP-PACS and JLQCD collaborations reported a result using $\mathcal{O}(a)$ improved Wilson fermions:

$$
\begin{aligned}
& m_{s}=91_{-6}^{+15} \mathrm{MeV} \\
& m_{l}=3.5_{-0.3}^{+0.6} \mathrm{MeV} .
\end{aligned}
$$

The two results are very compatible, giving necessary evidence that the results of lattice calculations are not dependent on the quark method.

Golden quantities and the CKM matrix elements. Most of the results discussed so far are for a particularly simple kind of quantity for lattice QCD: stable mesons (that is, ones that only decay weakly and not hadronically), in processes with a single meson present at a time. These are golden quantities for lattice QCD, with uncertainties that are smaller and easier to understand than for most quantities. Although this is a restricted set, many of the most important tasks of lattice gauge theory can be accomplished with quantities of this type. In particular, almost all of the CMK matrix elements and quark masses can be determined with lattice calculations in this category.

CKM matrix elements are measured in decay processes in which a quark of one flavor turns into a quark of another flavor. Fig. 7 illustrates $B$ meson "semileptonic" decay, that is, decay into two leptons plus one or more hadrons. In the experimentally observed process, a $B$ meson decays into two leptons, for example the electron and a neutrino, plus hadrons (labeled $X)$, for example a pion. The complicated strong interactions of gluons with quark-antiquark pairs responsible for confining the valence $b$ and $\bar{u}$ quarks in the $B$ meson is represented schematically in the figure by the curly red lines (gluons) and green circles (quark=antiquark pairs). The experimental rate depends on a QCD amplitude, which must be supplied by lattice QCD, and on the CKM matrix element $V_{u b}$, which is the coupling between an "up" quark $(u)$ and a "bottom" quark (b). Purely leptonic decays, such as a pion decaying into an electron plus a neutrino, are parameterized by decay constants such as $f_{\pi}$. Pion leptonic decay depends 
Table I. The Cabibbo-Kobayashi-Maskawa matrix elements, with particle processes by which they can be measured.

$$
\left(\begin{array}{ccc}
V_{u d} & V_{u s} & V_{u b} \\
f_{\pi} & f_{K} & f_{B} \\
& K \rightarrow \pi l \nu & B \rightarrow \pi l \nu \\
& & \\
V_{c d} & V_{c s} & V_{c b} \\
f_{D} & f_{D_{s}} & \\
D \rightarrow \pi l \nu & D \rightarrow K l \nu & B \rightarrow D l \nu \\
& & \\
V_{t d} & V_{t s} & V_{t b} \\
<B_{d} \mid \overline{B_{d}}> & <B_{s} \mid \overline{B_{s}}> & -
\end{array}\right)
$$

on the QCD amplitude $f_{\pi}$ and on $V_{u d}$, the CKM matrix element connecting up and down quarks. The amplitudes for mesons like $K, B$, and $B_{s}$ to mix with their antiparticles, $\bar{K}$, $\bar{B}, \overline{B_{s}}$, are proportional to other combinations of CKM matrix elements. In all, eight of the nine CKM matrix elements can be determined from relatively simple lattice QCD calculations combined with experiment, as shown in blue in Table I.

Semileptonic decays. In semileptonic decay, the shape of the decay amplitude as a function of the momentum of the decay products is predicted by lattice QCD and can be measured in experiment. Fig. 8 shows the form factor that parameterizes the decay amplitude for $D \rightarrow K l \nu$ semileptonic decay as a function of $t$, the square of the four-momentum transfered to the leptons, $l$ and $\nu$. The green points are lattice QCD predictions, the blue

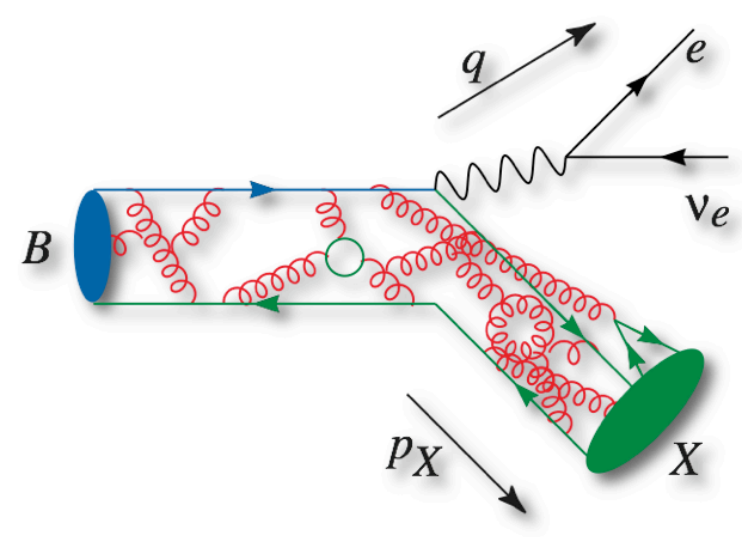

Fig. 7. The decay of a $B$ meson into leptons plus hadrons $X$, where $X$ might be a pion or a $D$ meson. ${ }^{19)}$ 
points are from the experiment of the Focus collaboration which appeared after the lattice predictions. ${ }^{20-22)}$ As can be seen, the agreement is excellent. Having tested the calculation by verifying that the predicted shape is correct, by comparing the normalization between theory and experiment, the CKM matrix element describing the quark coupling (in this case, $V_{c s}$ ) may be obtained.

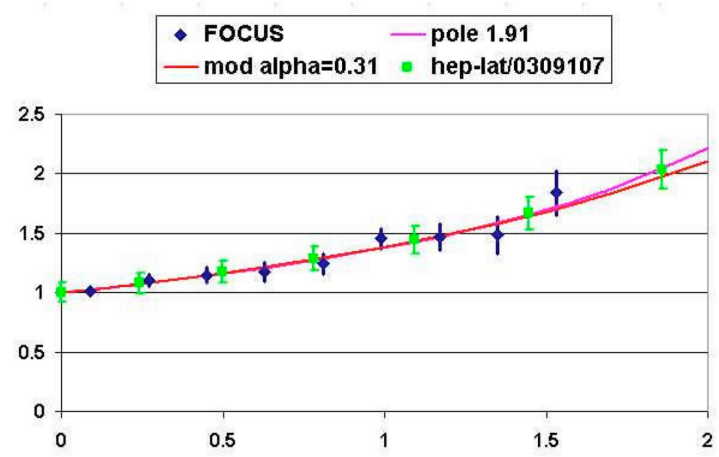

Fig. 8. Shape of the form factor describing the semileptonic decay $D \rightarrow K l \nu$, as a function of the momentum transfer $t=q^{2}$. The predicted shape (green circles) agrees well with the observed shape (blue diamonds). (Color online.)

$B \bar{B}$ and $B_{s} \bar{B}_{s}$ mixing. To illustrate the challenge ahead, consider the $\rho-\eta$ plane, shown in Fig. 9. In the Standard Model, the CKM matrix may be parameterized by four parameters, two of which are called $\rho$ and $\eta$. $\rho$ and $\eta$ have the form $\rho-i \eta \propto V_{u b}$. By determining these parameters in many different ways, one can test whether or not consistent results are obtained. Inconsistent results would be a signal of contributions to quark mixing from Beyondthe-Standard-Model theories, rather from the Standard Model alone.

$\rho$ and $\eta$ parameterize the $\mathrm{CP}$ violation in the Standard Model. CP is a symmetry relating the properties of particles to those of their antiparticles. Understanding the source of CP violation in nature is key to understanding the abundance of matter over antimatter in the visible universe. The plot is one of the most famous graphs in particle physics at the moment, and reducing its uncertainties is an important goal of particle physics.

The plot shows the bounds on the $\rho-\eta$ arising from various physical processes, with the small red circle illustrating the combined bound. Several of the uncertainties in the plot arise from estimates of the uncertainties in lattice QCD calculations. For example, the bounds in the dark green curves, labeled $\epsilon_{K}$, arise from measuring the mixing between $K$ mesons and their antiparticles, analyzed with lattice QCD. Similarly, the bounds in the yellow circles, labeled $\delta m_{d}$, arise from $B \bar{B}$ mixing. The orange circles show the constraint arising from the combination $B \bar{B}$ and $B_{s} \bar{B}_{s}$ mixing from before (Fig. 9) and after (Fig. 10) the discovery of $B_{s} \bar{B}_{s}$ mixing at the Tevatron last year. These constraints are only possible due to the 


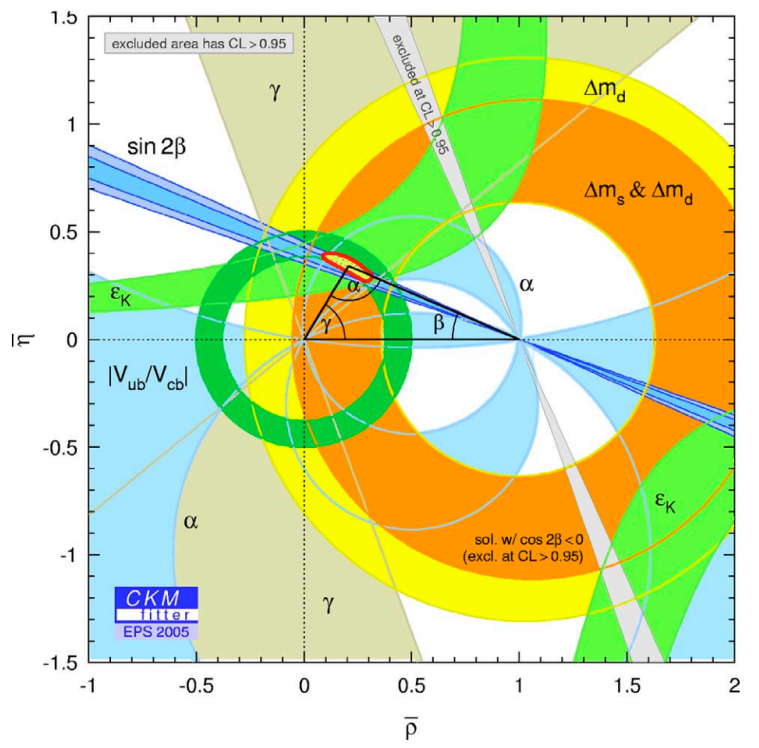

Fig. 9. Current bounds on $\rho$ and $\eta$, which parameterize CP violation in the CKM matrix. ${ }^{24)}$ The plot shows the status before the discovery of $B_{s} \bar{B}_{s}$ mixing at the Tevatron.

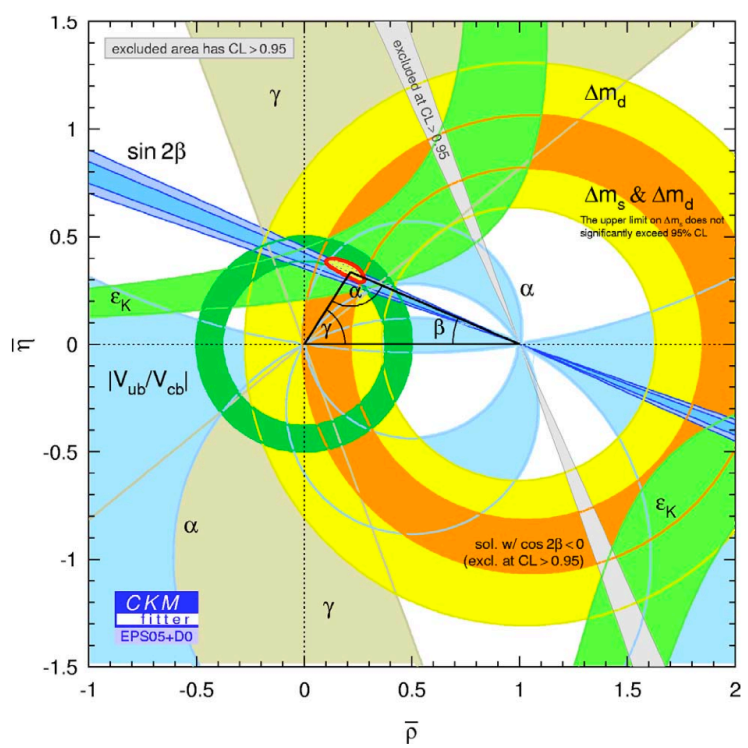

Fig. 10. Current bounds on $\rho$ and $\eta$, which parameterize CP violation in the CKM matrix. ${ }^{24)}$ The plot shows the status after the discovery of $B_{s} \bar{B}_{s}$ mixing at the Tevatron.

existence of good lattice gauge theory calculations. The experimental errors on the mixings that have been measured are of order $1 \%$. The 10 or $20 \%$ uncertainties in the quantities shown in the graph are estimates of the uncertainties of lattice calculations. The current round of calculations aims at reducing these to something of order $5 \%$. Clearly, to profit fully from the experiments that have been done, one needs to aim at lattice uncertainties of around $1 \%$, So challenging work remains ahead for lattice gauge theorists if the experimental results are to 
be fully exploited.

Decay constants To show that accuracies of $1-2 \%$ in this type of calculation are not an unreasonable goal, we can consider a new paper from HPQCD on decay constants (which parameterize the amplitudes for decay of a meson into a pair of leptons). They employ several improvements over previous calculations. Most importantly, they use a staggered fermion actions for the quarks that is more highly improved than previously (HISQ, or Highly Improved Staggered Quarks ${ }^{25)}$ ). They employ several other improvements to reduce the size of the uncertainties. Their results are: ${ }^{26)}$

$$
\begin{aligned}
f_{D} & =241(3) \mathrm{MeV} \\
f_{D_{s}} & =208(4) \mathrm{MeV} \\
f_{D_{s}} / f_{D} & =1.162(9), \\
f_{K} / f_{\pi} & =1.189(7) .
\end{aligned}
$$

The accuracies for the $D$ and $D_{s}$ decay constants are a factor of 4-5 improved over previous results ${ }^{27)}$ an impressive step forward.

Fig. 11 shows their results as a function of light quark mass, extrapolated down to the physical light quark limit, and compared there with experiment (black lines). In the case of the $D$ and $D_{s}$ decay constants, the theory calculations have moved quite a bit beyond experiment in accuracy (in contrast to the reverse situation in $B \bar{B}$ and $B_{s} \bar{B}_{s}$ mixing). The challenge now for lattice calculations is to extend this level of accuracy to many quantities.

\section{The future}

I have emphasized a small set of well-done quantities that have a strong connection to particle physics experiment. However, the current reach of lattice QCD is much broader than this. It is possible to study processes with multiple hadrons present at the same time, although more difficult than for single-hadron processes. The case of $K \rightarrow \pi \pi$ has been worked out very clearly. ${ }^{28)}$ Lattice calculations can investigate QCD in the realms of high temperatures and potentially of high densities that are of interest in neutron stars and the early universe. ${ }^{29)}$ Well-developed investigations of nuclear structure are underway. ${ }^{30)}$

Calculations continue to become more and more powerful through improved methods, better algorithms, and more powerful computers. This is allowing us to improve the precision of existing calculations, to verify that all fermion formulations give the same answers, and to extend our reach to new QCD quantities.

More exciting times could await lattice gauge theory in Beyond-the-Standard-Model physics, depending on what is discovered at the LHC. Such new physics could present a variety of challenges. For theories that are like QCD, but with larger numbers of colors or flavors, the same methods that are proving successful for QCD can be used. For simple su- 


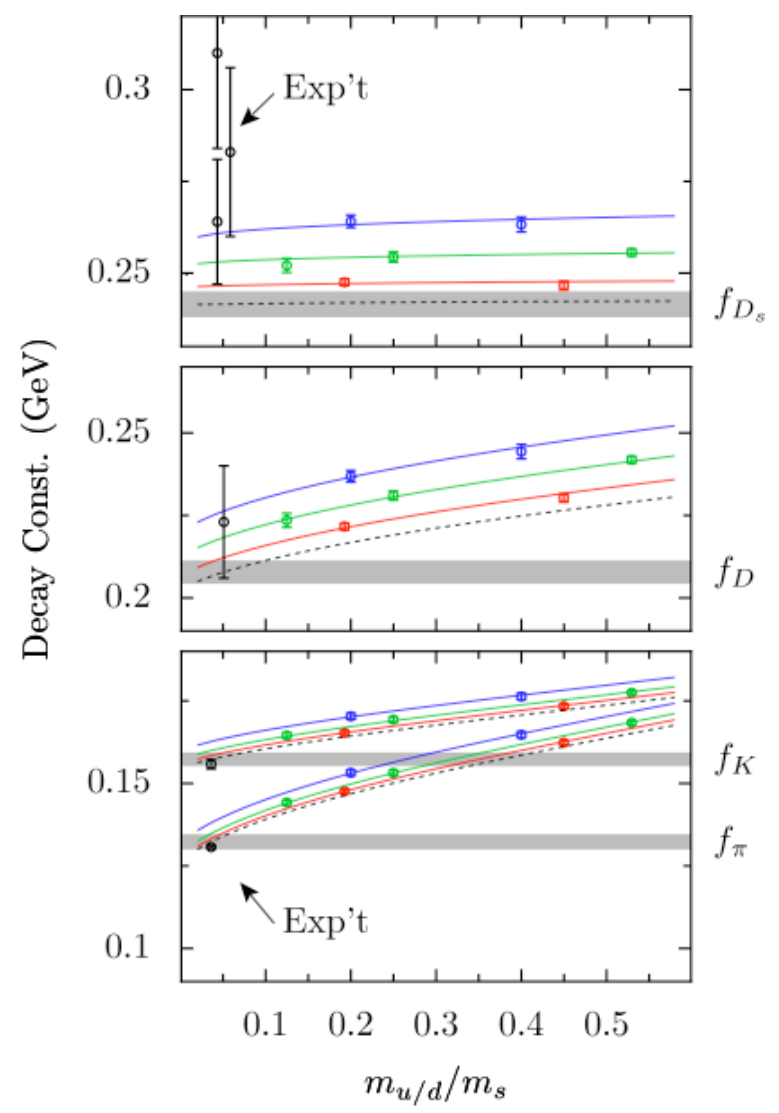

Fig. 11. The decay constants of the $D_{s}, D, K$, and $\pi$ mesons, as a function of the light quark mass. When the results are extrapolated to the physical light quark masses (black dashed lines), the results agree well with experiment (black circles at the left of the graphs). For the $D$ and $D_{s}$ mesons, the theory results are much more accurate than the experimental results. (Color online.)

persymmetric theories, promising methods are under development for their solutions. ${ }^{31)}$ For other conceivable new theories, major algorithmic advances will be required, for example in the very interesting case of theories in which right and left handed quarks do not come in pairs with the same color charge. The era of the LHC and Beyond-the-Standard-Model physics is likely to prove as eventful for lattice gauge theory as the current one has been. 


\section{References}

1) T. Wettig: "Performance of machines for lattice QCD simulations," PoS LAT2005 (2006) 019 [arXiv:hep-lat/0509103].

2) M. Luscher and P. Weisz: "Efficient Numerical Techniques For Perturbative Lattice Gauge Theory Computations," Nucl. Phys. B 266 (1986) 309.

3) S. Naik: "On-Shell Improved Lattice Action For QCD With Susskind Fermions And Asymptotic Freedom Scale," Nucl. Phys. B 316 (1989) 238;

4) D. Toussaint and K. Orginos [MILC Collaboration]: "Tests of improved Kogut-Susskind fermion actions," Nucl. Phys. Proc. Suppl. 73 (1999) 909 [arXiv:hep-lat/9809148];

5) G. P. Lepage: "Flavor-symmetry restoration and Symanzik improvement for staggered quarks," Phys. Rev. D 59 (1999) 074502 [arXiv:hep-lat/9809157].

6) S. R. Sharpe: "Rooted staggered fermions: Good, bad or ugly?," PoS LAT2006 (2006) 022 [arXiv:hep-lat/0610094].

7) M. Luscher: "Lattice QCD with light Wilson quarks," PoS LAT2005 (2006) 002 [arXiv:heplat/0509152].

8) M. J. Peardon and J. Sexton [TrinLat Collaboration]: "Multiple molecular dynamics time scales in hybrid Monte Carlo fermion simulations," Nucl. Phys. Proc. Suppl. 119 (2003) 985 [arXiv:heplat/0209037].

9) M. Hasenbusch: "Speeding up the Hybrid-Monte-Carlo algorithm for dynamical fermions," Phys. Lett. B 519 (2001) 177 [arXiv:hep-lat/0107019].

10) D. B. Kaplan: "A Method for simulating chiral fermions on the lattice," Phys. Lett. B 288 (1992) 342 [arXiv:hep-lat/9206013].

11) H. Neuberger: "Exactly massless quarks on the lattice," Phys. Lett. B $4 \mathbf{1 7}$ (1998) 141 [arXiv:heplat/9707022].

12) C. T. H. Davies et al. [Fermilab, HPQCD, MILC, UKQCD Collaborations]: "High-precision lattice QCD confronts experiment," Phys. Rev. Lett. 92 (2004) 022001 [arXiv:hep-lat/0304004].

13) I. F. Allison, C. T. H. Davies, A. Gray, A. S. Kronfeld, P. B. Mackenzie and J. N. Simone [HPQCD Collaboration]: "Mass of the B/c meson in three-flavor lattice QCD," Phys. Rev. Lett. 94 (2005) 172001 [arXiv:hep-lat/0411027].

14) W. M. Yao et al. [Particle Data Group]: "Review of particle physics," J. Phys. G 33 (2006) 1.

15) Q. Mason et al. [HPQCD Collaboration]: "Accurate determinations of alpha(s) from realistic lattice QCD," Phys. Rev. Lett. 95 (2005) 052002 [arXiv:hep-lat/0503005].

16) C. Aubin et al. [HPQCD, MILC Collaborations]: "First determination of the strange and light quark masses from full lattice QCD," Phys. Rev. D 70 (2004) 031504 [arXiv:hep-lat/0405022].

17) C. Bernard et al. [MILC Collaboration]: "Update on the physics of light pseudoscalar mesons," arXiv:hep-lat/0609053.

18) T. Ishikawa et al. [JLQCD Collaboration]: "Light quark masses from unquenched lattice QCD," arXiv:0704.1937 [hep-lat].

19) Graphic from talk of M. Luke at CKM 2005.

20) M. Okamoto et al.: "Semileptonic D $\rightarrow$ pi / K and B $\rightarrow$ pi / D decays in 2+1 flavor lattice QCD," Nucl. Phys. Proc. Suppl. 140 (2005) 461 [arXiv:hep-lat/0409116].

21) C. Aubin et al. [Fermilab Lattice Collaboration]: "Semileptonic decays of D mesons in three-flavor 
J. Phys. Soc. Jpn.

lattice QCD," Phys. Rev. Lett. 94 (2005) 011601 [arXiv:hep-ph/0408306].

22) For a recent review of heavy quark physics on the lattice, see T. Onogi: "Heavy flavor physics from lattice QCD," PoS LAT2006 (2006) 017 [arXiv:hep-lat/0610115].

23) M. Ciuchini, E. Franco, F. Parodi, V. Lubicz, L. Silvestrini and A. Stocchi: "Unitarity triangle analysis in the standard model and sensitivity to new physics," eConf C0304052 (2003) WG306 [arXiv:hep-ph/0307195].

24) Talk of D. Buchholz: Flavor Physics and CP Violation Conference (FPCP 2006), Vancouver, 9-12 Apr 2006.

25) E. Follana et al. [HPQCD Collaboration]: "Highly improved staggered quarks on the lattice, with applications to charm physics," Phys. Rev. D 75 (2007) 054502 [arXiv:hep-lat/0610092].

26) E. Follana, C. T. H. Davies, G. P. Lepage and J. Shigemitsu: "High Precision determination of the pi, K, D and Ds decay constants from lattice QCD," arXiv:0706.1726 [hep-lat].

27) C. Aubin et al.: "Charmed meson decay constants in three-flavor lattice QCD," Phys. Rev. Lett. 95 (2005) 122002 [arXiv:hep-lat/0506030].

28) L. Lellouch and M. Luscher: "Weak transition matrix elements from finite-volume correlation functions," Commun. Math. Phys. 219 (2001) 31 [arXiv:hep-lat/0003023].

29) For recent reviews, see U. M. Heller: "Recent progress in finite temperature lattice QCD," PoS LAT2006 (2006) 011 [arXiv:hep-lat/0610114]; C. Schmidt: "Lattice QCD at finite density," PoS LAT2006 (2006) 021 [arXiv:hep-lat/0610116].

30) K. Orginos: PoS LAT2006 (2006) 018.

31) J. Giedt: "Advances and applications of lattice supersymmetry," PoS LAT2006 (2006) 008 [arXiv:hep-lat/0701006]. 